\title{
STATUS OF THE PLASMA WAKEFIELD ACCELERATION EXPERIMENT AT THE STANFORD LINEAR ACCELERATOR CENTER*
}

\author{
P. Muggli ${ }^{*}$, M.J. Hogan ${ }^{\dagger}$, B.E. Blue ${ }^{\S}$, C. O’Connell ${ }^{\dagger}$, R.H. Siemann ${ }^{\dagger}$, D. Walz ${ }^{\dagger}$, R. Assmann ${ }^{\dagger}$, C.E. Clayton ${ }^{\S}$, F.- \\ S. Wang ${ }^{\S}$ \\ "University of Southern California, Los Angeles CA 90039, USA \\ ${ }^{\dagger}$ Stanford Linear Accelerator Center, Stanford CA 94309, USA \\ ${ }^{\S}$ University of California, Los Angeles CA 90095, USA \\ ${ }^{+}$CERN, Geneva, Switzerland
} J. Decker ${ }^{\dagger}$, E.S. Dodd ${ }^{\S}$, R.H. Iverson ${ }^{\dagger}$, C. Joshi ${ }^{\S}$, T.C. Katsouleas ${ }^{*}$, S. Lee ${ }^{*}$, K.A. Marsh ${ }^{\S}$, W.B. Mori ${ }^{\S}$, P. Raimondi ${ }^{\dagger}$,

\begin{abstract}
A plasma wakefield acceleration experiment is conducted at the Stanford Linear Accelerator Center. This experiment addresses the issues relevant to a meter-long plasma accelerator module in the context of a high-energy accelerator.
\end{abstract}

\section{INTRODUCTION}

Plasmas can sustain very large amplitude plasma waves. These waves can be excited by charged particle bunches or by intense laser pulses. In the plasma wakefield accelerator (PWFA), the space charge fields of a relativistic particle bunch exerts a radial force on the electrons of a neutral plasma, and drive a large amplitude plasma wake at the electron plasma frequency $\omega_{p e}=\left(n_{e} e^{2} / \varepsilon_{0} m\right)^{1 / 2}$ where $n_{e}$ is the plasma electron density, $e$ and $m$ are the electron charge and rest mass, and $\varepsilon_{0}$ is the vacuum permeability. The plasma wake has a radial electric field that focuses or defocuses the particle bunch, and a longitudinal electric field that decelerates particles in the bunch. Under the appropriate conditions this field can accelerates particles of the same bunch or of a separate witness bunch.

In the linear theory valid for bunches with density $n_{b}$ smaller than $n_{e}$, the amplitude of the longitudinal electric field can be written as:[1]

$$
e E_{z}=240(\mathrm{MeV} / \mathrm{m})\left(\frac{N}{4 \times 10^{10}}\right)\left(\frac{0.6 \mathrm{~mm}}{\sigma_{z}}\right)^{2}
$$

where $N$ is the number of particles in the driving bunch, and $\sigma_{z}$ is the root-mean-square length of the Gaussian bunch. The maximum gradient is obtained with a $n_{e}$ such that $\lambda_{p e}=2 \pi c / \omega_{p e} \approx 4 \sigma_{z}$ This expression shows that with bunches typical of the Final Focus Test Beam of the Stanford Linear Accelerator Center (FFTB-SLAC, $N=2 \times 10^{10}$ particle $/$ bunch,$\left.\quad \sigma_{z}=0.6 \mathrm{~mm}\right), \quad$ accelerating gradients larger than $100 \mathrm{MeV} / \mathrm{m}$ can be generated. This expression also shows the most attractive feature of the PWFA, namely the scaling of the accelerating gradient with the inverse square of the bunch length: $e E_{z} \propto \sigma_{z}^{-2}$.
To increase the accelerating gradient the PWFA can be run in the non-linear or over-dense regime where $n_{b}>n_{e}$. Full 2-D or 3-D particle in cell (PIC) numerical simulations using the OSIRIS code [2] have shown that in this regime the $\sigma_{z}^{-2}$ scaling remains valid.[1] In this regime the head of the bunch expels all the plasma electrons from the bunch volume ("blow-out" regime), and leaves a pure ion column for the core of the bunch to propagate through. In the pure ion column, the radial electric field is given by:

$$
E_{r}(r)=\frac{1}{2} \frac{n_{e} e}{\varepsilon_{0}} r
$$

The ion column acts an aberration-free plasma lens on the electron bunch.

The main issues that have to be addressed experimentally to make a PWFA module attractive for high-energy physics are the following:

- The making of meter-long sources of homogeneous plasma with $n_{e}$ in the $10^{14}$ to $10^{16} \mathrm{~cm}^{3}$ range.

- The stable propagation (against the hose instability) of $\mathrm{GeV}$ particle beams through these plasmas.

- The control of the transverse dynamics of GeV particle beams. The multiple betatron oscillations of the beam envelope observed with unmatched beams can be suppressed by matching the beam to the plasma, or by using hollow plasma channels.

- The preservation of the beam emittance through nonideal plasmas.

- The acceleration of particles at rates in the $100 \mathrm{MeV} / \mathrm{m}$ to $\mathrm{GeV} / \mathrm{m}$.

- The application of the PWFA to positrons for which the physics of the beam/plasma interaction is qualitatively different than for electrons.

\section{EXPERIMENTAL SET-UP AT THE SLAC-FFTB}

A 1.4 meter long plasma source has been developed for the E-157/E-162 PWFA experiments at SLAC. It consists of a lithium (Li) heat-pipe oven [3] with a neutral density $n_{0}$ in the $2-4 \times 0^{15} \mathrm{~cm}^{3}$ range. The $\mathrm{Li}$ has an ionization

\footnotetext{
${ }^{*}$ This work is supported by the US DoE Grants No. DE-FG03-92ER40745, No. DE-AC03-76SF00515, No. DE-FG03-98DP00211, and No. DEFG03-92ER40727, and NSF grants No. ECS-9632735, and No. DMS-9722121. We would like to thank Dr. Peter Tsou of JPL for providing the aerogel.
} 
potential of $5.4 \mathrm{eV}$, and is ionized by an ultra-violet (uv) laser pulse with $6.45 \mathrm{eV}$ photons to values of $n_{e}$ up to $\approx 5 \times 10^{14} \mathrm{~cm}^{3}$. The plasma density is maintained approximately constant along the oven despite the uv absorption by focusing the laser beam along the $\mathrm{Li}$ column. The $\mathrm{Li}$ neutral/plasma column length $L$ is obtained from longitudinal temperature profiles. The line integrated neutral density $\left(n_{0} L\right)$ is measured using the hook method[4] and uv absorption. The line integrated plasma density $\left(n_{e} L\right)$ was measured by $\mathrm{CO}_{2}$ laser interferometry [4], and is routinely monitored using uv absorption and uv beam spot size measurements. The particle beam traverses a thin fused silica pellicle at $45^{\circ}$ placed about $57 \mathrm{~cm}$ upstream from the Li vapor column (Fig. 1). The uv ionizing laser beam is overlapped with the particle beam onto the pellicle and is reflected toward the Li vapor, thereby making the particle beam and the plasma co-linear. The particle beam size and shape is monitored bout $1 \mathrm{~m}$ upstream (US) and downstream (DS) of the plasma edges by imaging the optical transition radiation (OTR) emitted by the beam traversing thin titanium foils. The spatial resolution of the OTR images is $\approx 9 \mu \mathrm{m}$. After the plasma, the particle beam travels through a dispersive magnet, and $\mathrm{a} \approx 1 \mathrm{~mm}$ thick piece of aerogel located $\approx 12 \mathrm{~m}$ DS from the plasma exit. The Cerenkov light emitted in the aerogel is spilt and one half imaged onto a CCD camera, yielding a time-integrated image of the particle beam at that location. The other half of the Cerenkov light is split, one half rotated and delayed, and both halves are imaged onto the slit of a streak camera with a $1 \mathrm{ps}$ time resolution [5]. A single streak camera image thus yields both a time resolved spot size of the beam in the plane without dispersion (x-plane), and a time resolved energy spectrum of the beam in the dispersive plane of the magnet (y-plane). The dispersion in the aerogel plane is $10 \mathrm{~cm}$ corresponding to $285 \mathrm{MeV} / \mathrm{mm}$. The imaging resolution is about $\approx 100 \mu \mathrm{m}$ in both planes corresponding to $\approx 28.5 \mathrm{MeV}$ in the y-plane. The beam position at different locations along the beam line is monitored using beam position monitors (BPMs). Typical parameters for the beam entering the plasma are shown in Table 1.

Table 1: Margin Specifications

\begin{tabular}{|l|l|l|}
\hline Parameter & Symbol & Value \\
\hline Beam Energy & $E$ & $28.5 \mathrm{GeV}$ \\
\hline $\begin{array}{l}\text { Beam } \\
\text { Relativistic } \\
\text { Factor }\end{array}$ & $\gamma$ & 55686 \\
\hline $\begin{array}{l}\text { Number of e-, } \\
\text { e+ per bunch }\end{array}$ & $N$ & $2 \times 10^{10}$ \\
\hline Bunch Length & $\sigma_{z}$ & $0.6 \mathrm{~mm}$ \\
\hline Bunch Radius & $\sigma_{x}, \sigma_{y}$ & $70 \mu \mathrm{m}$ \\
\hline Bunch Density & $n_{b}$ & $4 \times 10^{14} \mathrm{~cm}^{-3}$ \\
\hline $\begin{array}{l}\text { Normalized } \\
\text { Beam } \\
\text { Emittance }\end{array}$ & $\varepsilon_{x N}$ & $5 \times 10^{-5} \mathrm{~m}-\mathrm{rad}$ \\
\hline & $\varepsilon_{v N}$ & $0.5 \times 10^{-5} \mathrm{~m}-\mathrm{rad}$ \\
\hline Plasma Density & $n_{e}$ & $0-2 \times 10^{14} \mathrm{~cm}^{-3}$ \\
\hline Plasma Length & $L$ & $1.4 \mathrm{~m}$ \\
\hline
\end{tabular}

\section{EXPERIMENTAL RESULTS WITH ELECTRONS}

\subsection{Beam focusing}

With the parameters of Table $1 n_{b}>n_{e}$, and the plasma electrons are blown-out of the beam volume at an early time in the bunch, and most of the bunch experiences the uniform focusing force of the pure ion column. The plasma acts on the electron bunch as a thick, aberrationfree, plasma lens. The focusing field at the beam radius $\left(r=\sigma_{r}=70 \mu \mathrm{m}\right)$ can be estimated from Eq. (2) and is of the order of $94 \mathrm{MV} / \mathrm{m}$ with $n_{p}=1.5 \times 10^{14} \mathrm{~cm}^{-3}$, corresponding to a focusing strength $B_{\theta} / \sigma_{r}=4.5 \mathrm{kT} / \mathrm{m}$. With this large focusing field, the bunch envelope experiences multiple betatron oscillations over the plasma length. At the highest plasma density reached $\left(n_{e} \approx 5 \times 10^{14} \mathrm{~cm}^{3}\right)$ the total phase advance acquired by the beam particles over the plasma length is of the order of $6 \pi$. The beam size cannot be measured within the plasma. Instead, the beam spot size is
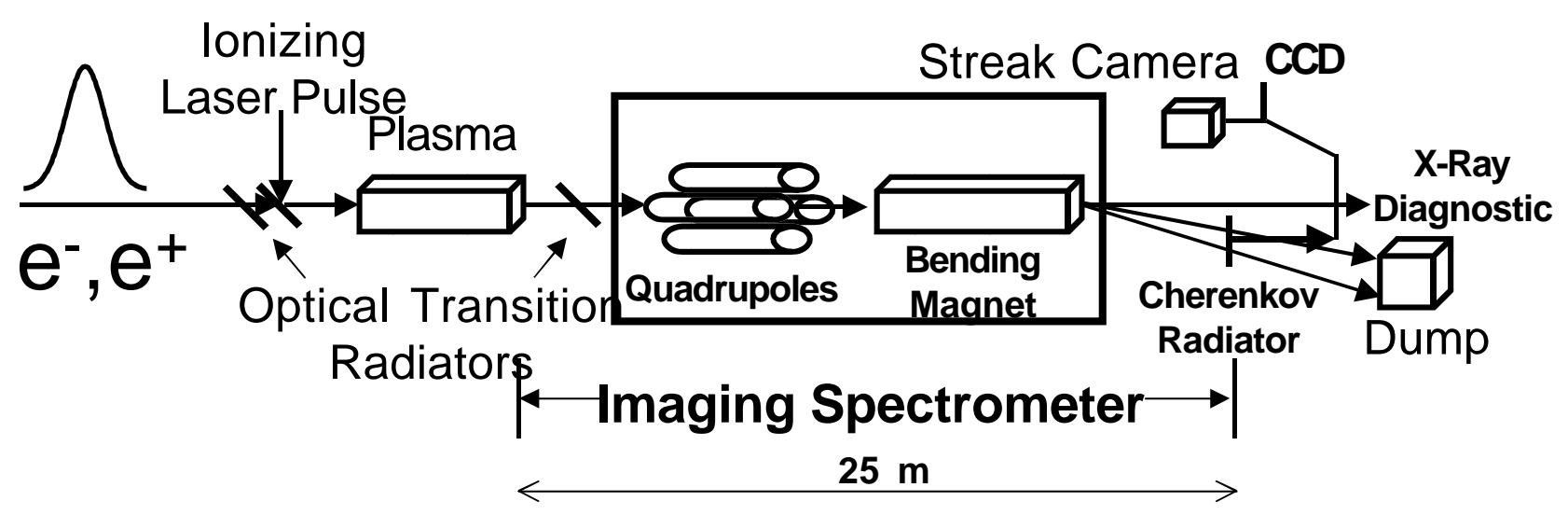

Figure 1: Experimental Set-Up 
measured at a location $\approx 1 \mathrm{~m}$ DS from the plasma exit as a function of $n_{e}$. The observed spot size oscillations are in good agreement with a simple beam envelope model describing the plasma as a thick lens [6] However, as the electrons in the head of the bunch expel the plasma electrons, they experience a focusing force that is weaker than in the "blow-out" regime. The focusing force thus increases from the beam head until the "blow-out" is reached. The associated dynamic focusing is also observed in the experiment.

\subsection{Tail oscillation/hose instability}

Transverse wake fields along the two mile long accelerator leading to the plasma source create a tail in the particle bunch. The strong transverse field of the plasma wake that focuses the bunch, can impart a transverse momentum to the bunch tail. The tail drifts ballistically from the plasma exit. The tail oscillates with twice the bunch envelope betatron period at any given plasma density. This tail oscillation is observed experimentally by measuring the beam center of charge position using BPMs and shows excellent agreement with the envelope model. The bunch tail acts as a seed for the beam hose instability. A simple linear theory for the hose instability of a short electron bunch in a preformed ion channel [7] predicts that the growth rate calculated with the E-157 experimental parameters is large enough for the hosing of the bunch to be observed. However, no significant hosing is observed in the experiment [8], or observed in 3-D PIC numerical simulations of the experiment.[9] This difference is attributed to the channel formation process happening both in the experiment and in the numerical simulations which decreases the growth of the hose instability.

\subsection{Synchrotron radiation}

As the electrons oscillate along their betatron motion, they emit $\mathrm{x}$-ray radiation in the $5-30 \mathrm{keV}$ energy range. This radiation has been observed previously in the microwave wavelength range and referred to as ion channel radiation (ICL) [10]. In the E-157 experiment the number of betatron oscillations experienced by the electrons is small $(<6)$, the radiation is in the spontaneous regime, and its total power scales as the square of the plasma density. The x-rays are emitted in a very narrow cone in the forward direction with a very high brightness [11].

\subsection{Beam steering/refraction}

When the bunch propagates in the homogeneous plasma the ion channel is axisymmetric, and the bunch does not experience an overall steering force. However, when the bunch crosses a plasma/vapor (or vacuum) boundary, ions are missing on the vapor side of the channel, and the ion channel becomes asymmetric. Therefore, the electron bunch emerging in vapor is attracted toward the plasma. This phenomena can be described a the refraction of the particle beam at the plasma/vapor boundary, much the same way a photon beams are refracted when crossing the boundary between two dielectrics. This phenomena results from the collective response of the very low-density plasma (as compared to liquid or solid densities at play in the photon beam case) to the bunch propagation. A nonlinear Snell's law can be derived [12] that describes qualitatively the behavior observed in the experiment [13]: at low incidence angle, the beam is internally reflected, at higher incidence angle, the core of the beam crosses the boundary and its trajectory is deflected toward the plasma. Single beam images $\approx 12 \mathrm{~m}$ downstream from the plasma show that the beam is spilt in two: the head of the beam forming the ion channel is not deflected at the plasma/vacuum boundary, whereas the back of the beam, that travels in the channel, is refracted at the boundary.

\subsection{Deceleration/Acceleration}

Magnetic quadrupoles have been recently added between the plasma source and the dispersive magnet. The optics allow for the imaging of the beam at the plasma exit onto the piece of aerogel where the beam energy measurement is performed [14]. The imaging system effectively removes the drift distance between the plasma and the aerogel. This prevents abeam tail drifting perpendicularly to the beam trajectory from appearing as an energy loss or gain signal on the streak camera images. In this case the contribution to the beam spot size in the $y$ plane, the dispersive plane of the magnet, is dominated by the natural energy spread of the beam or by energy gain resulting from the PWFA interaction and not by the beam divergence or the bunch tail. Preliminary results show unambiguous evidence of energy loss and gain by the beam particle. These results are presently analyzed and the results will be published elsewhere.

\section{EXPERIMENTAL RESULTS WITH POSITRONS}

Although the physics of the beam/plasma interaction is qualitatively different for electrons and positrons, positrons can be focused and accelerated by plasmas.

\subsection{Beam focusing}

In the case of positrons and electrons, the focusing of the beam results from its partial or total charge neutralization by the plasma. In the case of positrons, the plasma electrons are attracted into the positron bunch. However, unlike in the electron bunch case, there is no blow-out regime, and plasma electrons are continuously attracted from all radii smaller than $c / \omega_{p e}$, and arrive on axis at times that depend on their initial radius. The focusing of a positron beam is thus an essentially dynamic process both along the bunch and in the radial direction. Stronger focusing is expected for positrons than for electrons since in the case of positrons the charge density neutralizing the bunch electrostatic field can be larger than that of the plasma. Time integrated images of the positron beam downstream from the plasma show that the overall 
beam is focused by plasma densities in the $10^{12}-10^{13} \mathrm{~cm}^{-3}$ range. Detailed dynamic focusing of the positron bunch obtained from streak camera images will be published elsewhere [15].

\section{FUTURE WORK}

The beam transverse dynamics of an electron beam can be suppressed by matching the beam to the plasma $(1 / \beta=K)$. With the FFTB magnetic optics available for E162 Run 2 , beam sizes at the plasma entrance as small as $5 \mu \mathrm{m}$ can be reached at beam line focal point located near the plasma entrance. Attempts will be made to produce a beam with a radius of about $10 \mu \mathrm{m}$ at the plasma entrance, corresponding to the matched radius for a optimum plasma density for acceleration of about $1.5 \times 10^{14} \mathrm{~cm}^{-3}$.

The accelerating gradient for positrons in a homogeneous plasma is smaller than that of an electron beam, because plasma electrons are attracted into the positron bunch volume from all radii $<c / \omega_{p e}$. The plasma electrons arrival time on the beam axis can be "timed" by sending the positron beam in a hollow plasma channel of radius $\approx c / \omega_{p e}$. The accelerating gradient obtained in a hollow plasma channel is about twice that obtained in a homogeneous plasma [16]. Acceleration of positrons both in an homogeneous plasma and in a hollow plasma channel will be investigated at the end of 2001 .

Short electron bunches [17] will become available at SLAC at the end of 2002. A new plasma source will be developed to reach a plasma density of $\approx 6 \times 10^{15} \mathrm{~cm}^{-3}$, optimum for the acceleration of electron bunches with $\sigma_{z} \approx 100 \mu \mathrm{m}$. Excitation of accelerating gradients in excess of $4 \mathrm{GeV} / \mathrm{m}$ is expected with such parameters, leading to energy gains in excess of $1 \mathrm{GeV}$ over $30 \mathrm{~cm}$.

\section{REFERENCES}

[1] S. Lee et al., Phys. Rev. E 61(6), 7014 (2000).

[2] R. Hemker et al., Proceedings of the 1999 Particle Accelerator Conference, New York, NY, 3672, (1999).

[3] C.R. Vidal and J. Cooper, J. Appl. Phys. 40(8), 3370 (1969).

[4] P. Muggli et al., IEEE Trans. On Plasma Science 27(3), 791 (1999).

[5] M.J. Hogan et al, Physics of Plasmas 7(5), 2241 (2000).

[6] C.E. Clayton et al., submitted to Phys. Rev. Lett.

[7] D.H. Whittum, Journal of Physics D, 30(21), 2958 (1997).

[8] B. Blue et al., in preparation for Phys. Rev. Lett.

[9] E.S. Dodd et al., submitted to Phys. Rev. Lett. (2001).

[10] D.H. Whittum, et al., IEEE Trans. Plasma Sci. 21, 136, (1993).

[11] S. Wang et al., in preparation for Phys. Rev. Lett.

[12] T.C. Katsouleas et al., Nucl. Instr. and Meth. A 21, 161, (2000).

[13] P. Muggli et al., Nature 411, p.43 May 3 (2001).

[14] M.J. Hogan et al., these proceedings.

[15] M.J. Hogan et al., in preparation for Phys. Rev. Lett.

[16] S. Lee et al., submitted to Phys. Rev. Lett. (2001).

[17] J. Arthur et al., SLAC-PUB-8950, LCLS-TN-01-7, Aug 2001. 